\title{
Certain Flowers of Continued Fractions In the Garden of Generalized Lambert Series
}

\author{
Pankaj Srivastava (Corresponding author) \\ Department of Mathematics, Motilal Nehru National Institute of Technology \\ Allahabad 211 004, Uttar Pradesh, India \\ Tel: 941-561-6045Ｅ-mail: pankajs23@rediffmail.com \\ Mohan Rudravarapu \\ Department of Mathematics, Motilal Nehru National Institute of Technology \\ Allahabad 211 004, Uttar Pradesh, India
}

Tel: 880-890-9296, 944-124-7043 E-mail: mohanrudravarapu@hotmail.com

Received: March 5, 2012 Accepted: March 26, 2012 Online Published: May 28, 2012

doi:10.5539/jmr.v4n3p36 URL: http://dx.doi.org/10.5539/jmr.v4n3p36

\begin{abstract}
In this paper, an attempt has been made to establish certain results involving Lambert series and Continued fractions.

Keywords: Lambert series, Continued fractions, Rogers-Ramanujan identity

\section{Introduction}

Lambert series is a well known class of series in Analytic function theory and number theory. This series has been elegantly used in a variety of context of Ramanujan's research work. The dimension provided by Ramanujan inspired Andrews, G. E. and Berndt, B. C. (2005) to prove a lot of identities given by S. Ramanujan (Andrews, G. E. \& Berndt, B. C., 2005). Most of Ramanujan's unproved identities came in picture with their proofs nicely derived by Andrews, G. E. and Berndt, B. C. (2005) merely on the knowledge of Lambert series. The garden of Lambert series and generalized Lambert series enriched a lot due to the contribution of Agarwal, R. P. (1993), Denis, R. Y. (1985; 1984), Denis, R. Y., Singh, S. N., \& Singh, S. P. (2009), Bhargava, S. \& Somashekara, D. D. (1993), Naika, M. S. M. \& Dharmendra, B. N. (2008), Tachiya, Y. (2004), Srivastava, B. (2011; 2001), Assche, W. V. (2001), Cassens, P. \& Regan, F. (1970), Slater, L. J. (1952) and many others. We all are aware that continued fraction representations of basic hypergeometric series has given new direction for Ramanujan's work and mathematicians working in the field of continued fraction namely Agarwal, R. P. (1996), Denis, R. Y. (1985; 1983), Denis, R. Y., Singh, S. N., \& Singh, S. P. (2009; 2010), Denis, R. Y. \& Singh, S. N. (2000), Naika, M. S. M., Chandankumar, S., \& Sushan Bairy, K. (2012), Srivastava, P. (2008; 2011) and others have established hundreds of results for basic and bilateral basic hypergeometric functions and their continued fraction representations. In the present work we have used Generalized Lambert series and developed new continued fractions.
\end{abstract}

\section{Notations and Definitions}

For $a$ and $q$ real or complex and $|q|<1$, we shall have

$$
(a ; q)_{n}=\left\{\begin{array}{l}
1, \quad n=0 \\
(1-a)(1-a q) \ldots\left(1-a q^{n-1}\right), n=1,2,3 \ldots
\end{array}\right.
$$

We also define

$$
(a)_{\infty}=(a ; q)_{\infty}=\prod_{k=0}^{\infty}\left(1-a q^{k}\right), \text { for }|q|<1 .
$$

The infinite product diverges when $a \neq 0$.

Also

$$
\left(a_{1}, a_{2}, \ldots, a_{r} ; q\right)_{n}=\left(a_{1} ; q\right)_{n}\left(a_{2} ; q\right)_{n} \ldots\left(a_{r} ; q\right)_{n} .
$$




$$
[a ; q]_{n}=\frac{[a ; q]_{\infty}}{\left[a q^{n} ; q\right]_{\infty}} .
$$

An expression of the form $\frac{a_{0}}{b_{0}+} \frac{a_{1}}{b_{1}+\ldots}$ is said to be a continued fraction. The values of $a_{1}, a_{2}, a_{3}, \ldots$ and $b_{1}, b_{2}, b_{3}, \ldots$ can be either real or complex values. A finite simple continued fraction is a simple continued fraction with only a finite number of terms. An infinite simple continued fraction is a simple continued fraction with an infinite number of terms.

The series, $\sum_{n=1}^{\infty} a_{n} \frac{x^{n}}{1-x^{n}}$, considered by Lambert in connection with the convergence of power series is called Lambert series.If the series $\sum_{n=1}^{\infty} a_{n}$ converges, then Lambert series, $\sum_{n=1}^{\infty} a_{n} \frac{x^{n}}{1-x^{n}}$ converges for all values of $x$ except for $x= \pm 1$, otherwise it converges for those values of $x$, for which the series $\sum_{n=1}^{\infty} a_{n} x^{n}$ converges. A series of the form $\sum_{n=-\infty}^{n=\infty}(-1)^{\epsilon n} q^{\lambda n^{2}} R\left(q^{n}\right)$, where $\epsilon=0$ or $1, \lambda>0$, and $R\left(q^{n}\right)$ is rational function of $q^{n}$ is called generalized Lambert series.

We use following continued fractions for analysis in the present paper.

Rogers - Ramanujan continued fraction, $c(q)$ is given by

$$
\frac{1}{c(q)}=\frac{1}{1+} \frac{q}{1+} \frac{q^{2}}{1+} \frac{q^{3}}{1+} \frac{q^{4}}{1+\ldots}=\frac{\sum_{n=0}^{\infty} \frac{q^{n^{2}+n}}{[q ; q]_{n}}}{\sum_{n=0}^{\infty} \frac{q^{n^{2}}}{[q ; q]_{n}}}=\frac{\left[q ; q^{5}\right]_{\infty}\left[q^{4} ; q^{5}\right]_{\infty}}{\left[q^{2} ; q^{5}\right]_{\infty}\left[q^{3} ; q^{5}\right]_{\infty}}, \quad|q|<1
$$

(Andrews, G. E. \& Berndt, B. C., 2005, 10.4.1, p. 248).

$$
\frac{\left[q ; q^{6}\right]_{\infty}\left[q^{5} ; q^{6}\right]_{\infty}}{\left[q^{3} ; q^{6}\right]_{\infty}^{2}}=\frac{1}{1+} \frac{q+q^{2}}{1+} \frac{q^{2}+q^{4}}{1+} \frac{q^{3}+q^{6}}{1+\ldots},
$$

(Andrews, G. E. \& Berndt, B. C., 2005, 6.2.37, p. 154).

$$
\frac{\left[q ; q^{8}\right]_{\infty}\left[q^{7} ; q^{8}\right]_{\infty}}{\left[q^{3} ; q^{8}\right]_{\infty}\left[q^{5} ; q^{8}\right]_{\infty}}=\frac{1}{1+} \frac{q+q^{2}}{1+} \frac{q^{4}}{1+} \frac{q^{3}+q^{6}}{1+\ldots},
$$

(Andrews, G. E. \& Berndt, B. C., 2005, 6.2.38, p. 154).

$$
\frac{\left[q ; q^{2}\right]_{\infty}}{\left[q^{2} ; q^{4}\right]_{\infty}^{2}}=\frac{1}{1+} \frac{q}{1+} \frac{q+q^{2}}{1+} \frac{q^{3}}{1+} \frac{q^{2}+q^{4}}{1+\ldots},
$$

(Andrews, G. E. \& Berndt, B. C., 2005, 6.2.22, p. 150).

$$
\frac{\left[q^{3} ; q^{4}\right]_{\infty}}{\left[q ; q^{4}\right]_{\infty}}=\frac{1}{1-} \frac{q}{1+q^{2}-} \frac{q^{3}}{1+q^{4}-} \frac{q^{5}}{1+q^{6}-\ldots},
$$

(Andrews, G. E. \& Berndt, B. C., 2005, p. 156).

$$
\frac{\left[q^{2} ; q^{3}\right]_{\infty}}{\left[q ; q^{3}\right]_{\infty}}=\frac{1}{1-} \frac{q}{1+q-} \frac{q^{3}}{1+q^{2}-} \frac{q^{5}}{1+q^{3}-\ldots},
$$

(Andrews, G. E. \& Berndt, B. C., 2005, 8.1.1, p. 197).

The first remarkable result of Rogers - Ramanujan continued fraction involving Lambert series is given by

$$
\frac{1}{c^{3}(q)}=\frac{\sum_{n=-\infty}^{\infty} \frac{q^{2 n}}{1-q^{5 n+2}}}{\sum_{n=-\infty}^{\infty} \frac{q^{n}}{1-q^{5 n+1}}}=\frac{\sum_{n=0}^{\infty} q^{5 n^{2}+4 n} \frac{1+q^{5 n+2}}{1-q^{5 n+2}}-\sum_{n=0}^{\infty} q^{5 n^{2}+6 n+1} \frac{1+q^{5 n+3}}{1-q^{5 n+3}}}{\sum_{n=0}^{\infty} q^{5 n^{2}+2 n} \frac{1+q^{5 n+1}}{1-q^{5 n+1}}-\sum_{n=0}^{\infty} q^{5 n^{2}+8 n+3} \frac{1+q^{5 n+4}}{1-q^{5 n+4}}},
$$

(Andrews, G. E. \& Berndt, B. C., 2005, 4.4.1, 4.4.2, p. 117). 
Here $C(q)$ is called Ramanujan continued fraction with its value equal to $G(q) / H(q)$, and $G(q), H(q)$ are called Rogers - Ramanujan identities, which are as follows

$$
\begin{aligned}
& G(q)=\sum_{n=0}^{\infty} \frac{q^{n^{2}}}{[q ; q]_{n}}=\frac{1}{\left[q ; q^{5}\right]_{\infty}\left[q^{4} ; q^{5}\right]_{\infty}}, \\
& H(q)=\sum_{n=0}^{\infty} \frac{q^{n^{2}+n}}{[q ; q]_{n}}=\frac{1}{\left[q^{2} ; q^{5}\right]_{\infty}\left[q^{3} ; q^{5}\right]_{\infty}},
\end{aligned}
$$

(Andrews, G. E. \& Berndt, B. C., 2005, 4.3.3, 4.3.4, p. 114).

We also use the following identities for establishing our main results.

The Rogers-Fine identity is as follows

$$
\sum_{n=0}^{\infty} \frac{[\alpha ; q]_{n}}{[\beta: q]_{n}} z^{n}=\sum_{n=0}^{\infty} \frac{[\alpha ; q]_{n}[\alpha z q / \beta ; q]_{n} \beta^{n} z^{n} q^{n^{2}-n}\left(1-\alpha z q^{2 n}\right)}{[\beta ; q]_{n}[z ; q]_{n+1}},
$$

(Andrews, G. E. \& Berndt, B. C., 2005, 9.1.1, p. 223).

$$
\begin{aligned}
& \sum_{n=0}^{\infty} \frac{z^{n}}{\left(1-\alpha q^{n}\right)}=\sum_{n=0}^{\infty} \frac{(\alpha z)^{n} q^{n^{2}}\left(1-\alpha z q^{2 n}\right)}{\left(1-\alpha q^{n}\right)\left(1-z q^{n}\right)}, \\
& \sum_{n=0}^{\infty} \frac{q^{n j}}{\left(1-q^{k n+i}\right)}=\sum_{n=0}^{\infty} \frac{q^{k n^{2}+n(i+j)}\left(1-q^{2 k n+i+j}\right)}{\left(1-q^{k n+i}\right)\left(1-q^{k n+j}\right)},
\end{aligned}
$$

(Denis, R. Y., 1985, 2.2).

$$
\sum_{n=0}^{\infty} \frac{q^{n i}}{\left(1-q^{k n+i}\right)}=\sum_{n=0}^{\infty} \frac{q^{k n^{2}+2 n i}\left(1+q^{k n+i}\right)}{\left(1-q^{k n+i}\right)},
$$

(Denis, R. Y., Singh, S. N., \& Singh, S. P., 2009, 3.4).

$$
\sum_{n=-\infty}^{\infty} \frac{q^{n j}}{\left(1-q^{k n+i}\right)}=\frac{\left[q^{k} ; q^{k}\right]_{\infty}^{2}\left[q^{i+j} ; q^{k}\right]_{\infty}\left[q^{k-i-j} ; q^{k}\right]_{\infty}}{\left[q^{i} ; q^{k}\right]_{\infty}\left[q^{j} ; q^{k}\right]_{\infty}\left[q^{k-i} ; q^{k}\right]_{\infty}\left[q^{k-j} ; q^{k}\right]_{\infty}},
$$

(Denis, R. Y., Singh, S. N., \& Singh, S. P., 2009, 3.1).

$$
\sum_{n=0}^{\infty} \frac{(n+1)[q / \alpha ; q]_{n} \alpha^{n}}{[\beta ; q]_{n+1}}+\sum_{n=0}^{\infty} \frac{n[q / \beta ; q]_{n} \beta^{n}}{[\alpha ; q]_{n+1}}=\frac{[q ; q]_{\infty}^{3}[\alpha \beta ; q]_{\infty}}{[\alpha ; q]_{\infty}^{2}[\beta ; q]_{\infty}^{2}}
$$

(Bhargava, S. \& Somashekara, D. D., 1993, 2.3, p. 556).

$$
\sum_{n=0}^{\infty} \frac{(n+1)\left[q^{k-i} ; q^{k}\right]_{n} q^{i n}}{\left[q^{j} ; q^{k}\right]_{n+1}}+\sum_{n=0}^{\infty} \frac{n\left[q^{k-j} ; q^{k}\right]_{n} q^{j n}}{\left[q^{i} ; q^{k}\right]_{n+1}}=\frac{\left[q^{k} ; q^{k}\right]_{\infty}^{3}\left[q^{i+j} ; q^{k}\right]_{\infty}}{\left[q^{i} ; q^{k}\right]_{\infty}^{2}\left[q^{j} ; q^{k}\right]_{\infty}^{2}},
$$

(Denis, R. Y., Singh, S. N., \& Singh, S. P., 2009, 3.7).

$$
\begin{gathered}
{\left[q^{5} ; q^{5}\right]_{\infty}^{2} G(q)=\sum_{n=-\infty}^{\infty} \frac{q^{n}}{1-q^{5 n+2}},} \\
{\left[q^{5} ; q^{5}\right]_{\infty}^{2} H(q)=\sum_{n=-\infty}^{\infty} \frac{q^{3 n}}{1-q^{5 n+1}},} \\
{\left[q^{5} ; q^{5}\right]_{\infty}^{2} \frac{G^{2}(q)}{H(q)}=\sum_{n=-\infty}^{\infty} \frac{q^{n}}{1-q^{5 n+1}},} \\
{\left[q^{5} ; q^{5}\right]_{\infty}^{2} \frac{H^{2}(q)}{G(q)}=\sum_{n=-\infty}^{\infty} \frac{q^{2 n}}{1-q^{5 n+2}},}
\end{gathered}
$$




$$
\begin{gathered}
{\left[q^{5} ; q^{5}\right]_{\infty}^{2} G(q)=\sum_{n=-\infty}^{\infty} \frac{q^{2 n}}{1-q^{5 n+1}},} \\
{\left[q^{5} ; q^{5}\right]_{\infty}^{2} H(q)=\sum_{n=-\infty}^{\infty} \frac{q^{n}}{1-q^{5 n+3}},} \\
{\left[q^{5} ; q^{5}\right]_{\infty}^{2} \frac{G^{2}(q)}{H(q)}=\sum_{n=-\infty}^{\infty} \frac{q^{5 n^{2}+2 n}\left(1+q^{5 n+1}\right)}{1-q^{5 n+1}},} \\
{\left[q^{5} ; q^{5}\right]_{\infty}^{2} \frac{H^{2}(q)}{G(q)}=\sum_{n=-\infty}^{\infty} \frac{q^{5 n^{2}+4 n}\left(1+q^{5 n+2}\right)}{1-q^{5 n+2}},} \\
{\left[q^{5} ; q^{5}\right]_{\infty}^{2} G(q)=\sum_{n=-\infty}^{\infty} \frac{q^{4 n}}{1-q^{10 n+1}},} \\
{\left[q^{5} ; q^{5}\right]_{\infty}^{2} H(q)=\sum_{n=-\infty}^{\infty} \frac{q^{2 n}}{1-q^{10 n+3}},}
\end{gathered}
$$

(Andrews, G. E. \& Berndt, B. C., 2005, (21)...(30)).

\section{Main Results}

We established the following main results

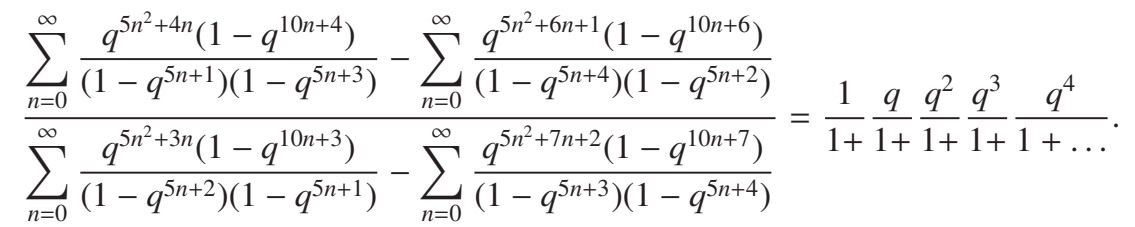

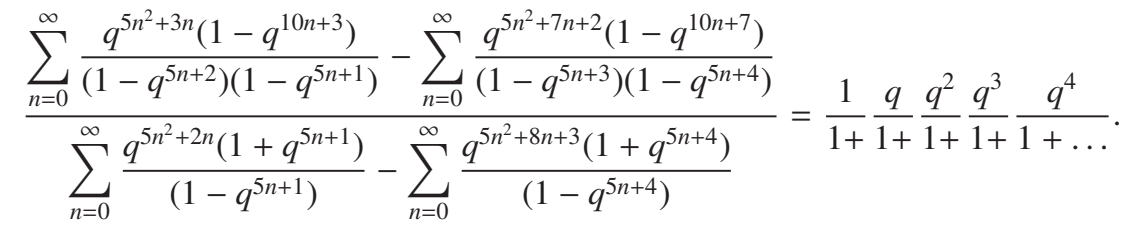

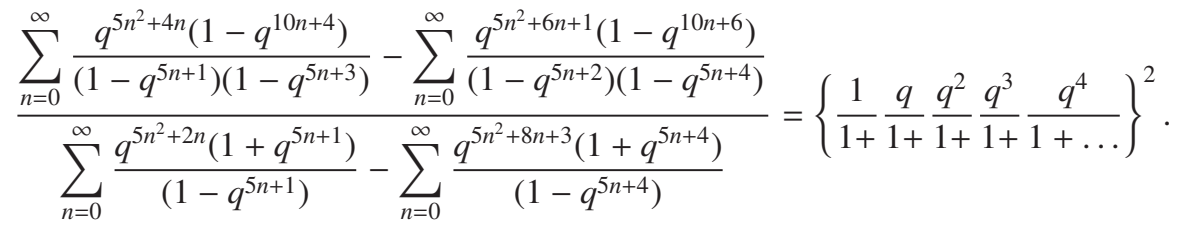

$$
\begin{aligned}
& \frac{\sum_{n=0}^{\infty} \frac{q^{5 n^{2}+4 n}\left(1+q^{5 n+2}\right)}{\left(1-q^{5 n+2}\right)}-\sum_{n=0}^{\infty} \frac{q^{5 n^{2}+6 n+1}\left(1+q^{5 n+3}\right)}{\left(1-q^{5 n+3}\right)}}{\sum_{n=0}^{\infty} \frac{q^{5 n^{2}+3 n}\left(1-q^{10 n+3}\right)}{\left(1-q^{5 n+1}\right)\left(1-q^{5 n+2}\right)}-\sum_{n=0}^{\infty} \frac{q^{5 n^{2}+7 n+2}\left(1-q^{10 n+7}\right)}{\left(1-q^{5 n+4}\right)\left(1-q^{5 n+3}\right)}}=\left\{\frac{1}{1+} \frac{q}{1+} \frac{q^{2}}{1+} \frac{q^{3}}{1+} \frac{q^{4}}{1+\ldots}\right\}^{2} . \\
& \frac{\sum_{n=0}^{\infty} \frac{q^{5 n^{2}+4 n}\left(1+q^{5 n+2}\right)}{\left(1-q^{5 n+2}\right)}-\sum_{n=0}^{\infty} \frac{q^{5 n^{2}+6 n+1}\left(1+q^{5 n+3}\right)}{\left(1-q^{5 n+3}\right)}}{\sum_{n=0}^{\infty} \frac{q^{5 n^{2}+4 n}\left(1-q^{10 n+4}\right)}{\left(1-q^{5 n+3}\right)\left(1-q^{5 n+1}\right)}-\sum_{n=0}^{\infty} \frac{q^{5 n^{2}+6 n+1}\left(1-q^{10 n+6}\right)}{\left(1-q^{5 n+4}\right)\left(1-q^{5 n+2}\right)}}=\frac{1}{1+} \frac{q}{1+} \frac{q^{2}}{1+} \frac{q^{3}}{1+} \frac{q^{4}}{1+\ldots} .
\end{aligned}
$$

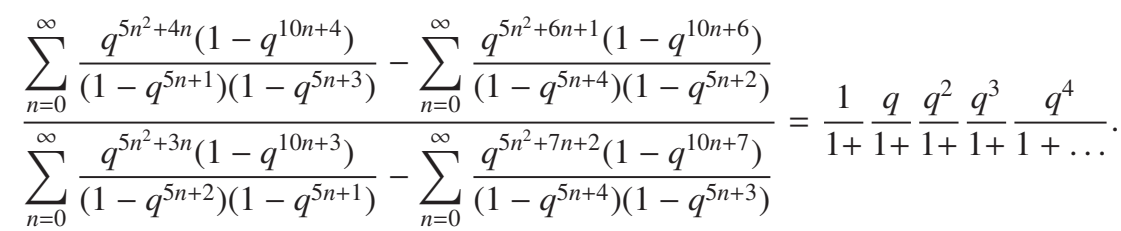




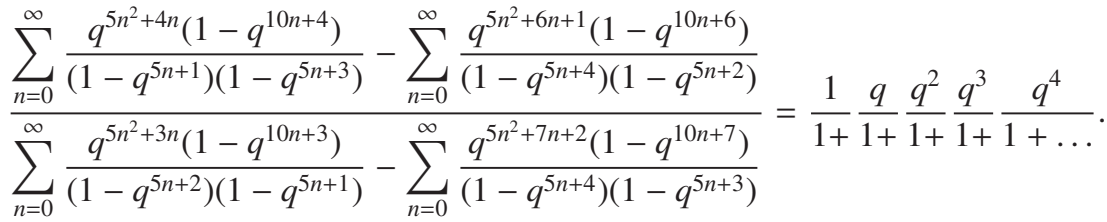

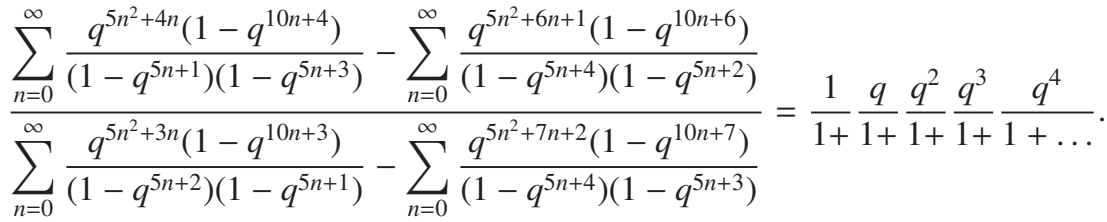

$$
\begin{aligned}
& \frac{\sum_{n=0}^{\infty} \frac{q^{5 n^{2}+4 n}\left(1+q^{5 n+2}\right)}{\left(1-q^{5 n+2}\right)}-\sum_{n=0}^{\infty} \frac{q^{5 n^{2}+6 n+1}\left(1+q^{5 n+3}\right)}{\left(1-q^{5 n+3}\right)}}{\sum_{n=0}^{\infty} \frac{q^{5 n^{2}+3 n}\left(1-q^{10 n+3}\right)}{\left(1-q^{5 n+1}\right)\left(1-q^{5 n+2}\right)}-\sum_{n=0}^{\infty} \frac{q^{5 n^{2}+7 n+2}\left(1-q^{10 n+7}\right)}{\left(1-q^{5 n+4}\right)\left(1-q^{5 n+3}\right)}}=\left\{\frac{1}{1+} \frac{q}{1+} \frac{q^{2}}{1+} \frac{q^{3}}{1+} \frac{q^{4}}{1+\ldots}\right\}^{2} . \\
& \frac{\sum_{n=0}^{\infty} \frac{q^{5 n^{2}+4 n}\left(1-q^{10 n+4}\right)}{\left(1-q^{5 n+3}\right)\left(1-q^{5 n+1}\right)}-\sum_{n=0}^{\infty} \frac{q^{5 n^{2}+6 n+1}\left(1-q^{10 n+6}\right)}{\left(1-q^{5 n+2}\right)\left(1-q^{5 n+4}\right)}}{\sum_{n=0}^{\infty} \frac{q^{5 n^{2}+2 n}\left(1+q^{5 n+1}\right)}{\left(1-q^{5 n+1}\right)}-\sum_{n=0}^{\infty} \frac{q^{5 n^{2}+8 n+3}\left(1+q^{5 n+4}\right)}{\left(1-q^{5 n+4}\right)}}=\left\{\frac{1}{1+} \frac{q}{1+} \frac{q^{2}}{1+} \frac{q^{3}}{1+\ldots}\right\}^{2} . \\
& \frac{\sum_{n=0}^{\infty} \frac{q^{5 n^{2}+4 n}\left(1-q^{10 n+4}\right)}{\left(1-q^{5 n+3}\right)\left(1-q^{5 n+1}\right)}-\sum_{n=0}^{\infty} \frac{q^{5 n^{2}+6 n+1}\left(1-q^{10 n+6}\right)}{\left(1-q^{5 n+2}\right)\left(1-q^{5 n+4}\right)}}{\sum_{n=0}^{\infty} \frac{q^{5 n^{2}+2 n}\left(1+q^{5 n+1}\right)}{\left(1-q^{5 n+1}\right)}-\sum_{n=0}^{\infty} \frac{q^{5 n^{2}+8 n+3}\left(1+q^{5 n+4}\right)}{\left(1-q^{5 n+4}\right)}}=\left\{\frac{1}{1+} \frac{q}{1+} \frac{q^{2}}{1+} \frac{q^{3}}{1+} \frac{q^{4}}{1+\ldots}\right\}^{2} . \\
& \frac{\sum_{n=0}^{\infty} \frac{q^{5 n^{2}+4 n}\left(1+q^{5 n+2}\right)}{\left(1-q^{5 n+2}\right)}-\sum_{n=0}^{\infty} \frac{q^{5 n^{2}+6 n+1}\left(1+q^{5 n+3}\right)}{\left(1-q^{5 n+3}\right)}}{\sum_{n=0}^{\infty} \frac{q^{5 n^{2}+3 n}\left(1-q^{10 n+3}\right)}{\left(1-q^{5 n+1}\right)\left(1-q^{5 n+2}\right)}-\sum_{n=0}^{\infty} \frac{q^{5 n^{2}+7 n+2}\left(1-q^{10 n+7}\right)}{\left(1-q^{5 n+4}\right)\left(1-q^{5 n+3}\right)}}=\left\{\frac{1}{1+} \frac{q}{1+} \frac{q^{2}}{1+} \frac{q^{3}}{1+} \frac{q^{4}}{1+\ldots}\right\}^{2} . \\
& \frac{\sum_{n=0}^{\infty} \frac{q^{5 n^{2}+4 n}\left(1+q^{5 n+2}\right)}{\left(1-q^{5 n+2}\right)}-\sum_{n=0}^{\infty} \frac{q^{5 n^{2}+6 n+1}\left(1+q^{5 n+3}\right)}{\left(1-q^{5 n+3}\right)}}{\sum_{n=0}^{\infty} \frac{q^{10 n^{2}+5 n}\left(1-q^{20 n+5}\right)}{\left(1-q^{10 n+1}\right)\left(1-q^{10 n+4}\right)}-\sum_{n=0}^{\infty} \frac{q^{10 n^{2}+15 n+5}\left(1-q^{20 n+15}\right)}{\left(1-q^{10 n+9}\right)\left(1-q^{10 n+6}\right)}}=\left\{\frac{1}{1+} \frac{q}{1+} \frac{q^{2}}{1+} \frac{q^{3}}{1+} \frac{q^{4}}{1+\ldots}\right\}^{2} . \\
& \frac{\sum_{n=0}^{\infty} \frac{q^{10 n^{2}+5 n}\left(1-q^{20 n+5}\right)}{\left(1-q^{10 n+2}\right)\left(1-q^{10 n+3}\right)}-\sum_{n=0}^{\infty} \frac{q^{10 n^{2}+15 n+5}\left(1-q^{20 n+15}\right)}{\left(1-q^{10 n+7}\right)\left(1-q^{10 n+8}\right)}}{\sum_{n=0}^{\infty} \frac{q^{5 n^{2}+2 n}\left(1+q^{5 n+1}\right)}{\left(1-q^{5 n+1}\right)}-\sum_{n=0}^{\infty} \frac{q^{5 n^{2}+8 n+3}\left(1+q^{5 n+4}\right)}{\left(1-q^{5 n+4}\right)}}=\left\{\frac{1}{1+} \frac{q}{1+} \frac{q^{2}}{1+} \frac{q^{3}}{1+} \frac{q^{4}}{1+\ldots}\right\}^{2} \\
& \frac{\sum_{n=0}^{\infty} \frac{q^{5 n^{2}+4 n}\left(1+q^{5 n+2}\right)}{1-q^{5 n+2}}-\sum_{n=0}^{\infty} \frac{q^{5 n^{2}+6 n}\left(1+q^{5 n+3}\right)}{1-q^{5 n+3}}}{\sum_{n=0}^{\infty} \frac{q^{5 n^{2}+6 n}\left(1-q^{10 n+6}\right)}{\left(1-q^{5 n+2}\right)\left(1-q^{5 n+4}\right)}-\sum_{n=0}^{\infty} \frac{q^{5 n^{2}+4 n-1}\left(1-q^{10 n+4}\right)}{\left(1-q^{5 n+1}\right)\left(1-q^{5 n+3}\right)}}=-q\left\{\frac{1}{1+} \frac{q}{1+} \frac{q^{2}}{1+} \frac{q^{3}}{1+} \frac{q^{4}}{1+\ldots}\right\} . \\
& \frac{\sum_{n=0}^{\infty} \frac{q^{5 n^{2}+6 n}\left(1-q^{10 n+6}\right)}{\left(1-q^{5 n+2}\right)\left(1-q^{5 n+4}\right)}-\sum_{n=0}^{\infty} \frac{q^{5 n^{2}+4 n-1}\left(1-q^{10 n+4}\right)}{\left(1-q^{5 n+1}\right)\left(1-q^{5 n+3}\right)}}{\sum_{n=0}^{\infty} \frac{q^{5 n^{2}+2 n}\left(1+q^{5 n+1}\right)}{1-q^{5 n+1}}-\sum_{n=0}^{\infty} \frac{q^{5 n^{2}+8 n+3}\left(1+q^{5 n+4}\right)}{1-q^{5 n+4}}}=-\frac{1}{q}\left\{\frac{1}{1+} \frac{q}{1+} \frac{q^{2}}{1+} \frac{q^{3}}{1+} \frac{q^{4}}{1+\ldots}\right\}^{2} .
\end{aligned}
$$




$$
\begin{aligned}
& \frac{\sum_{n=0}^{\infty} \frac{q^{6 n^{2}+7 n}\left(1-q^{12 n+7}\right)}{\left(1-q^{6 n+4}\right)\left(1-q^{6 n+3}\right)}-\sum_{n=0}^{\infty} \frac{q^{6 n^{2}+5 n-1}\left(1-q^{12 n+5}\right)}{\left(1-q^{6 n+3}\right)\left(1-q^{6 n+2}\right)}}{\sum_{n=0}^{\infty} \frac{q^{6 n^{2}+3 n}\left(1-q^{12 n+3}\right)}{\left(1-q^{6 n+1}\right)\left(1-q^{6 n+2}\right)}-\sum_{n=0}^{\infty} \frac{q^{6 n^{2}+9 n}\left(1-q^{12 n+9}\right)}{\left(1-q^{6 n+4}\right)\left(1-q^{6 n+5}\right)}}=-\frac{1}{q}\left\{\frac{1}{1+} \frac{q+q^{2}}{1+} \frac{q^{2}+q^{4}}{1+} \frac{q^{3}+q^{6}}{1+\ldots}\right\}^{2} . \\
& \frac{\sum_{n=0}^{\infty} \frac{q^{6 n^{2}+7 n}\left(1-q^{12 n+7}\right)}{\left(1-q^{6 n+4}\right)\left(1-q^{6 n+3}\right)}-\sum_{n=0}^{\infty} \frac{q^{6 n^{2}+5 n}\left(1-q^{12 n+5}\right)}{\left(1-q^{6 n+3}\right)\left(1-q^{6 n+2}\right)}}{\sum_{n=0}^{\infty} \frac{q^{6 n^{2}+4 n}\left(1+q^{6 n+2}\right)}{1-q^{6 n+2}}-\sum_{n=0}^{\infty} \frac{q^{6 n^{2}+8 n-2}\left(1+q^{6 n+4}\right)}{1-q^{6 n+4}}}=-\frac{1}{q}\left\{\frac{1}{1+} \frac{q+q^{2}}{1+} \frac{q^{2}+q^{4}}{1+} \frac{q^{3}+q^{6}}{1+\ldots}\right\} . \\
& \frac{\sum_{n=0}^{\infty} \frac{q^{8 n^{2}+7 n}\left(1-q^{16 n+7}\right)}{\left(1-q^{8 n+5}\right)\left(1-q^{8 n+2}\right)}-\sum_{n=0}^{\infty} \frac{q^{8 n^{2}+9 n+1}\left(1-q^{16 n+9}\right)}{\left(1-q^{8 n+3}\right)\left(1-q^{8 n+6}\right)}}{\sum_{n=0}^{\infty} \frac{q^{8 n^{2}+5 n}\left(1-q^{16 n+5}\right)}{\left(1-q^{8 n+2}\right)\left(1-q^{8 n+3}\right)}-\sum_{n=0}^{\infty} \frac{q^{8 n^{2}+11 n+3}\left(1-q^{16 n+11}\right)}{\left(1-q^{8 n+6}\right)\left(1-q^{8 n+5}\right)}}=\left\{\frac{1}{1+} \frac{q+q^{2}}{1+} \frac{q^{4}}{1+} \frac{q^{3}+q^{6}}{1+\ldots}\right\} . \\
& \frac{\sum_{n=0}^{\infty} \frac{q^{8 n^{2}+9 n}\left(1-q^{16 n+9}\right)}{\left(1-q^{8 n+7}\right)\left(1-q^{8 n+2}\right)}-\sum_{n=0}^{\infty} \frac{q^{8 n^{2}+7 n-1}\left(1-q^{16 n+7}\right)}{\left(1-q^{8 n+1}\right)\left(1-q^{8 n+6}\right)}}{\sum_{n=0}^{\infty} \frac{q^{8 n^{2}+5 n}\left(1-q^{16 n+5}\right)}{\left(1-q^{8 n+4}\right)\left(1-q^{8 n+1}\right)}-\sum_{n=0}^{\infty} \frac{q^{8 n^{2}+11 n+3}\left(1-q^{16 n+11}\right)}{\left(1-q^{8 n+4}\right)\left(1-q^{8 n+7}\right)}} \\
& =-\frac{1}{q}\left\{\frac{1}{1+} \frac{q+q^{2}}{1+} \frac{q^{4}}{1+} \frac{q^{3}+q^{6}}{1+\ldots}\right\}\left\{1+\frac{q^{2}}{1+} \frac{q^{2}+q^{4}}{1+} \frac{q^{6}}{1+} \frac{q^{4}+q^{8}}{1+\ldots}\right\} . \\
& \frac{\sum_{n=0}^{\infty} \frac{q^{4 n^{2}+5 n}\left(1-q^{8 n+5}\right)}{\left(1-q^{4 n+3}\right)\left(1-q^{4 n+2}\right)}-\sum_{n=0}^{\infty} \frac{q^{4 n^{2}+3 n}\left(1-q^{8 n+3}\right)}{\left(1-q^{4 n+1}\right)\left(1-q^{4 n+2}\right)}}{\sum_{n=0}^{\infty} \frac{q^{4 n^{2}+2 n}\left(1+q^{4 n+1}\right)}{1-q^{4 n+1}}-\sum_{n=0}^{\infty} \frac{q^{4 n^{2}+6 n+2}\left(1+q^{4 n+3}\right)}{1-q^{4 n+3}}}=-\frac{1}{q}\left\{\frac{1}{1+} \frac{q}{1+} \frac{q+q^{2}}{1+} \frac{q^{3}}{1+\ldots}\right\}^{2} . \\
& \frac{\sum_{n=0}^{\infty} \frac{q^{4 n^{2}+3 n}\left(1-q^{8 n+3}\right)}{\left(1-q^{4 n+2}\right)\left(1-q^{4 n+1}\right)}-\sum_{n=0}^{\infty} \frac{q^{4 n^{2}+5 n+1}\left(1-q^{8 n+5}\right)}{\left(1-q^{4 n+2}\right)\left(1-q^{4 n+3}\right)}}{\sum_{n=0}^{\infty} \frac{q^{4 n^{2}+2 n}\left(1+q^{4 n+1}\right)}{1-q^{4 n+1}}-\sum_{n=0}^{\infty} \frac{q^{4 n^{2}+6 n+2}\left(1+q^{4 n+3}\right)}{1-q^{4 n+3}}}=\left\{\frac{1}{1+} \frac{q}{1+} \frac{q+q^{2}}{1+} \frac{q^{3}}{1+} \frac{q^{2}+q^{4}}{1+\ldots}\right\}^{2} . \\
& \frac{\sum_{n=0}^{\infty} \frac{(2 n+1)\left[q^{4} ; q^{6}\right]_{n} q^{2 n}}{\left[q^{2} ; q^{6}\right]_{n+1}}}{\sum_{n=0}^{\infty} \frac{(2 n+1)\left[q^{2} ; q^{6}\right]_{n} q^{4 n}}{\left[q^{4} ; q^{6}\right]_{n+1}}}=\left(1-q^{2}\right)\left\{\frac{1}{1-} \frac{q^{2}}{1+q^{2}-} \frac{q^{6}}{1+q^{4}-} \frac{q^{10}}{1+q^{6}-\ldots}\right\}^{5} . \\
& \frac{\sum_{n=0}^{\infty} \frac{(2 n+1)\left[q^{6} ; q^{8}\right]_{n} q^{2 n}}{\left[q^{2} ; q^{8}\right]_{n+1}}}{\sum_{n=0}^{\infty} \frac{(2 n+1)\left[q^{2} ; q^{8}\right]_{n} q^{6 n}}{\left[q^{6} ; q^{8}\right]_{n+1}}}=\left(1-q^{4}\right)\left\{\frac{1}{1-\frac{q^{2}}{1+q^{4}-}} \frac{q^{6}}{1+q^{8}-} \frac{q^{10}}{1+q^{12}-\ldots}\right\}^{4} .
\end{aligned}
$$

\section{Proof of Main Results}

Proof (31)-(44):

As an illustration, we shall prove (31). 
From (21) and (22) we have

$$
\frac{H(q)}{G(q)}=\frac{\sum_{n=-\infty}^{\infty} \frac{q^{3 n}}{1-q^{5 n+1}}}{\sum_{n=-\infty}^{\infty} \frac{q^{n}}{1-q^{5 n+2}}}=\frac{\sum_{n=0}^{\infty} \frac{q^{3 n}}{1-q^{5 n+1}}-\sum_{n=0}^{\infty} \frac{q^{2 n+1}}{1-q^{5 n+4}}}{\sum_{n=0}^{\infty} \frac{q^{n}}{1-q^{5 n+2}}-\sum_{n=0}^{\infty} \frac{q^{4 n+2}}{1-q^{5 n+3}}}
$$

Making use of (5) and (16) in (55) and after simplification we obtain (31).

Proceeding in the same way and using the results (5), (16), (17) and (21)-(30), one can establish the results (32)(44).

Proof of (45)-(54):

In order to prove results (45) to (54), we proceed as follows:

As an illustration, we shall prove (47) and (50).

Proof of (47) is as follows Taking $i=3, j=4, k=6$ in (18) we get

$$
\sum_{n=-\infty}^{\infty} \frac{q^{4 n}}{1-q^{6 n+3}}=\frac{\left[q^{6} ; q^{6}\right]_{\infty}^{2}\left[q^{7} ; q^{6}\right]_{\infty}\left[q^{-1} ; q^{6}\right]_{\infty}}{\left[q^{3} ; q^{6}\right]_{\infty}^{2}\left[q^{4} ; q^{6}\right]_{\infty}\left[q^{2} ; q^{6}\right]_{\infty}}
$$

Again setting $i=2, j=1, k=6$ in (18) we get

$$
\sum_{n=-\infty}^{\infty} \frac{q^{n}}{1-q^{6 n+2}}=\frac{\left[q^{6} ; q^{6}\right]_{\infty}^{2}\left[q^{3} ; q^{6}\right]_{\infty}^{2}}{\left[q^{2} ; q^{6}\right]_{\infty}\left[q ; q^{6}\right]_{\infty}\left[q^{4} ; q^{6}\right]_{\infty}\left[q^{5} ; q^{6}\right]_{\infty}}
$$

Now taking the ratio of (56) and (57) and making use of (16) for the assigned values of $i, j$ and $k$, we get

$$
\frac{\sum_{n=0}^{\infty} \frac{q^{6 n^{2}+7 n}\left(1-q^{12 n+7}\right)}{\left(1-q^{6 n+4}\right)\left(1-q^{6 n+3}\right)}-\sum_{n=0}^{\infty} \frac{q^{6 n^{2}+5 n-1}\left(1-q^{12 n+5}\right)}{\left(1-q^{6 n+3}\right)\left(1-q^{6 n+2}\right)}}{\sum_{n=0}^{\infty} \frac{q^{6 n^{2}+3 n}\left(1-q^{12 n+3}\right)}{\left(1-q^{6 n+1}\right)\left(1-q^{6 n+2}\right)}-\sum_{n=0}^{\infty} \frac{q^{6 n^{2}+9 n}\left(1-q^{12 n+9}\right)}{\left(1-q^{6 n+4}\right)\left(1-q^{6 n+5}\right)}}=\frac{\left[q^{7} ; q^{6}\right]_{\infty}\left[q^{-1} ; q^{6}\right]_{\infty}\left[q ; q^{6}\right]_{\infty}^{2}\left[q^{5} ; q^{6}\right]_{\infty}^{2}}{\left[q q_{\infty}\left[q^{5} ; q^{6}\right]_{\infty}\right.}
$$

Finally making use of (6) in (58) and after some calculation, we get (47).

In order to prove (50) we proceed as follows consider $i=2, j=7, k=8$ in (18) we get

$$
\sum_{n=-\infty}^{\infty} \frac{q^{7 n}}{1-q^{8 n+2}}=\frac{\left[q^{8} ; q^{8}\right]_{\infty}^{2}\left[q^{9} ; q^{8}\right]_{\infty}\left[q^{-1} ; q^{8}\right]_{\infty}}{\left[q^{2} ; q^{8}\right]_{\infty}\left[q^{7} ; q^{8}\right]_{\infty}\left[q^{6} ; q^{8}\right]_{\infty}\left[q ; q^{8}\right]_{\infty}}
$$

Further taking $i=1, j=4, k=8$ in (18) we obtain

$$
\sum_{n=-\infty}^{\infty} \frac{q^{4 n}}{1-q^{8 n+1}}=\frac{\left[q^{8} ; q^{8}\right]_{\infty}^{2}\left[q^{5} ; q^{8}\right]_{\infty}\left[q^{3} ; q^{8}\right]_{\infty}}{\left[q ; q^{8}\right]_{\infty}\left[q^{4} ; q^{8}\right]_{\infty}\left[q^{7} ; q^{8}\right]_{\infty}\left[q^{4} ; q^{8}\right]_{\infty}}
$$

Now taking the ratio of (59) and (60) and making use of (16) for the assigned values of $i, j$ and $k$, we get

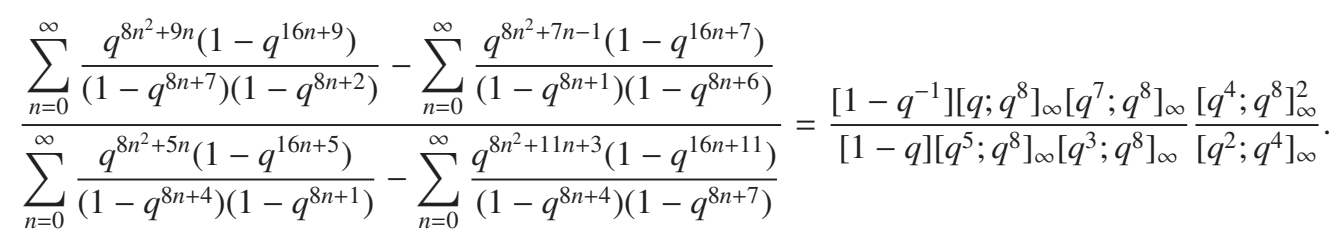

Now making use of (7) and (8) in (61), and after some calculation we obtain (50).

For suitable selection of values for $i, j$ and $k$ and using the same process for the results (5) to (10) and (16) to (20), one can establish the results (45), (46), (48), (49) and (51) to (54). 


\section{Conclusion}

The object of this article has been to show the effective applications of generalized Lambert series in obtaining a diverse variety of continued fractions.

\section{Acknowledgment}

The authors would like to thank the editor and reviewer for their valuable suggestions and comments.

\section{References}

Agarwal, R. P. (1993). Lambert series and Ramanujan. Proceedings Mathematical Sciences, 103(3), 269-293. http://dx.doi.org/ 10.1007/BF02866991

Agarwal, R. P. (1996). Resonance of Ramanujan's Mathematics, volume-II. New Delhi: New Age International Limited.

Andrews, G. E., \& Berndt, B. C. (2005). Ramanujan's Lost Notebook, Part 1. New York: Springer.

Assche, W. V. (2001). Little q-Legendre polynomials and irrationality of certain Lambert series. Katholieke Universiteit and Georgia Institute of Technology.

Bhargava, S., \& Somashekara, D. D. (1993). Some eta-function identities deduced from Ramanujan's ${ }_{1} \psi_{1}$ summation. J. Math. Anal. Appl, 176(2), 554-560. http://dx.doi.org/10.1006/jmaa.1993.1231

Cassens, P., \& Regan, F. (1970). On generalized Lambert summability. Commentationes Mathematicae Universitatis Carolinae, 11(4), 829-839.

Denis, R. Y. (1985). On certain q-series and continued fraction identities. Math. Student, 53, 243-248.

Denis, R. Y. (1984). (issued in 1988). On Generalisations of certain q-series results of Ramanujan. Math. Student, 52, 47-58.

Denis, R. Y., Singh, S. N., \& Singh, S. P. (2009). On Lambert series and continued fractions. Italian Journal of Pure and Applied Mathematics, N-26, 9-16.

Denis, R. Y., Singh, S. N., \& Singh, S. P. (2010). On certain continued fraction representations of poly basic series. South East Asian J. Math and Math. Sci., 8(2), 25-33.

Denis, R. Y. (1983). On certain q-series and continued fractions. The Math. Student, 44, 70-76.

Denis, R. Y., \& Singh, S. N. (2000). Hypergeometric functions and continued fractions. Far East J. Math. Sci., 2(3), 385-400.

Naika, M. S. M., \& Dharmendra, B. N. (2008). On Some new general theorems for the explicit evaluations of Ramanujan's remarkable product of Theta functions. The Ramanujan Journal, 15(3), 349-366. http://dx.doi.org/10.1007/s11139-007-9081-1

Naika, M. S. M., Chandankumar, S., \& Sushan Bairy, K. (2012). On Some New Identities for Ramanujans Cubic Continued Fraction. Int. J. Contemp. Math. Sciences, 7(20), 953-962.

Slater, L. J. (1952). Further identities of the Rogers-Ramanujan type. Proc. London Math. Soc, 54(2), 147-167. http://dx.doi.org/10.1112/plms/s2-54.2.147

Srivastava, B. (2001). Some q-identities associated with Ramanujan,s continued fraction. Kodai Math. Journal, 24, 36-41. http://dx.doi.org/10.2996/kmj/1106157293

Srivastava, B. (2011). On Modular equations and Lambert series for a continued fraction of Ramanujan. Bulletin of Mathematical Analysis and Applications, 3(1), 148-155.

Srivastava, P. (2008). Certain continued fractions for quotients of two ${ }_{3} \psi_{3}$ series. Proc. Nat. Acad. Sci. India, 78(A) IV, 327-330.

Srivastava, P. (2011). Resonance of continued fractions related to ${ }_{2} \psi_{2}$ basic bilateral hypergeometric series. Kyungpook Math. J., 51, 419-427. http://dx.doi.org/10.5666/KMJ.2011.51.4.419

Tachiya, Y. (2004). Irrationality of certain Lambert series. Tokyo Journal of Mathematics, 27(1), 75-85. http://dx.doi.org/10.3836/tjm/1244208475 